\title{
Superior immune reconstitution using Treg-expanded donor cells versus PTCy treatment in preclinical HSCT models
}

\author{
Dietlinde Wolf, ${ }^{1}$ Cameron S. Bader, ${ }^{2}$ Henry Barreras, ${ }^{2}$ Sabrina Copsel, ${ }^{2}$ Brent J. Pfeiffer, ${ }^{3}$ \\ Casey 0. Lightbourn, ${ }^{2}$ Norman H. Altman, ${ }^{4}$ Krishna V. Komanduri, ${ }^{1,2,5}$ and Robert B. Levy,2,5 \\ ${ }^{1}$ Sylvester Comprehensive Cancer Center, ${ }^{2}$ Department of Microbiology \& Immunology, ${ }^{3}$ Department of Pediatrics, \\ ${ }^{4}$ Department of Pathology and Laboratory Medicine, and ${ }^{5}$ Department of Medicine, Miller School of Medicine, University of \\ Miami, Miami, Florida, USA.
}

\begin{abstract}
Posttransplant cyclophosphamide (PTCy) has been found to be effective in ameliorating acute graft-versus-host disease (CVHD) in patients following allogeneic hematopoietic stem cell transplantation (aHSCT). Adoptive transfer of high numbers of donor Tregs in experimental aHSCT has shown promise as a therapeutic modality for GVHD regulation. We recently described a strategy for in vivo Treg expansion targeting two receptors: TNFRSF25 and CD25. To date, there have been no direct comparisons between the use of PTCy and Tregs regarding outcome and immune reconstitution within identical groups of transplanted mice. Here, we assessed these two strategies and found both decreased clinical CVHD and improved survival long term. However, recipients transplanted with Treg-expanded donor cells (TrED) exhibited less weight loss early after HSCT. Additionally, TrED recipients demonstrated less thymic damage, significantly more recent thymic emigrants, and more rapid lymphoid engraftment. Three months after HSCT, PTCy-treated and TrED recipients showed tolerance to $\mathrm{F} 1$ skin allografts and comparable immune function. Overall, TrED was found superior to PTCy with regard to weight loss early after transplant and initial lymphoid engraftment. Based on these findings, we speculate that morbidity and mortality after transplant could be diminished following TrED transplant into aHSCT recipients, and, therefore, that TrED could provide a promising clinical strategy for CVHD prophylaxis.
\end{abstract}

Authorship note: KVK and RBL are co-senior authors.

Conflict of interest: RBL is a consultant for Allergan, HEAT Biologics, and Pelican Therapeutics and is on the scientific advisory board of HEAT Biologics.

License: Copyright 2018, American Society for Clinical Investigation.

Submitted: April 30, 2018

Accepted: August 30, 2018

Published: October 18, 2018

Reference information: JCI Insight. 2018;3(20):e121717. https://doi.org/10.1172/jici. insight.121717.

\section{Introduction}

Development of graft-versus-host disease (GVHD) remains a major obstacle to long-term transplant success following allogeneic hematopoietic stem cell transplantation (aHSCT) (1-3). While advances in GVHD prophylaxis based on the calcineurin or MTOR inhibitors have facilitated safe matched related and unrelated donor HSCT, limits in the success of these approaches, including delayed immune reconstitution, significantly limit the broader application of aHSCT to other diseases, including autoimmune disorders. Within the past 10 years, the use of short-term posttransplant cyclophosphamide (PTCy) following clinical aHSCT on days 3 and 4 has proven an extremely effective strategy for acute GVHD prophylaxis and has facilitated a significant improvement and expansion in the success of mismatched HSCT, including using haploidentical and MUD donors (4-6).

One of the most critical challenges in HSCT is the development of therapies that suppress GVHD while facilitating enhanced immune reconstitution relative to existing strategies that involve long-term maintenance immunosuppression (2). $\mathrm{CD}^{+} \mathrm{FoxP}^{+}$Tregs have been clearly proven capable, in murine models and early human clinical studies, of reducing alloreactive anti-donor and anti-host $\mathrm{T}$ cell responses after allo-HSCT (7-13). However, native frequencies of Tregs in donor grafts are insufficient to facilitate transplantation across mismatched barriers; consequently, it has become clear that therapeutic application of these cells will require significant enrichment of Tregs from their basal frequencies. Several clinical investigations have shown promise in the use of ex vivo-expanded Tregs for GVHD amelioration (11-13), and experimental studies continue to evaluate how to expand and enhance Treg function to maximize their capacity to induce immune transplant tolerance and ameliorate GVHD (14-16). We have recently described a novel strategy that markedly expands Tregs in vivo following signaling by TNFRSF25 and CD25 receptors (10). Transplantation of cells from donor mice that have undergone this transient Treg 
expansion demonstrated effective amelioration of GVHD following MHC-mismatched and -matched aHSCT and maintain graft-versus-leukemia responses critical for limitation of relapse (10).

Although short-term effects of cyclophosphamide on immune function are well defined $(17,18)$, there have been limited studies involving long-term immune reconstitution following PTCy (19, 20). Reports have noted that rates of infection and relapse have not been significantly increased in contrast to historical approaches to haploidentical HSCT that were based on ex vivo T cell depletion as well as compared with contemporaneous haploidentical, MRD, and MUD HSCTs (5, 21-24). To date, there have been no direct comparisons between the use of PTCy and Tregs regarding outcome and immune reconstitution within identical groups of transplanted mice. It has been clear for approximately 20 years that thymopoiesis persists into late adulthood in humans, albeit variably, and contributes significantly to lymphoid homeostasis in healthy and immunocompromised adults (25-27). Prior clinical studies in humans have demonstrated that earlier recovery of thymopoiesis in adults (28) and children (29) is associated with improved recovery of naive and functional T cells critical for the control of after HSCT infections.

To compare several aspects of immune reconstitution, we examined recipients of experimental aHSCT receiving either standard PTCy (i.e., using high-dose cyclophosphamide administered day +3 and +4 ) or transplant with donor cells containing high levels of in vivo-expanded Tregs and observed that (a) both treatment strategies ameliorate GVHD involving MHC-mismatched as well as MHC-matched allogeneic models; (b) both treatment strategies lead to effective immune function later after HSCT; (c) early after HSCT, recipients of Treg-expanded donor (TrED) murine grafts experience significantly less weight loss and other clinical signs of GVHD versus those receiving PTCy; and (d) early thymic reconstitution is markedly superior in recipients of TrED grafts. These findings suggest that the strategy of in vivo donor Treg expansion for GVHD prophylaxis is accompanied by more rapid recovery of thymopoiesis and that this strategy may promote earlier recovery of a diverse and functional $\mathrm{T}$ cell repertoire during the early period following HSCT.

\section{Results}

Relative to PTCy treatment, use of TrED donors results in lower early weight loss but comparable long-term outcomes following minor-and major-mismatched HSCT. To directly compare the capacity of TrED with the use of PTCy in the regulation of GVHD, two established experimental models of HSCT were used for analysis. First, a complete MHC-disparate HSCT was performed using B6 $\left(\mathrm{H} 2^{\mathrm{b}}\right)$ donors transplanted into BALB/c $\left(\mathrm{H} 2^{\mathrm{d}}\right)$ recipients (Figure 1). T cells were obtained from donor B6-FIR mice following expansion of their Treg compartment or from unexpanded animals. Tregs were expanded by targeting the TNFRSF25 and CD25 receptors using an agonistic fusion protein (TL1A-Ig) and low-dose IL-2, as previously described (10). Donor spleen cells analyzed prior to transplant regularly demonstrated $50 \%$ of the CD4 T cells expressed FoxP3, as anticipated (Supplemental Figure 1; supplemental material available online with this article; https://doi.org/10.1172/jci.insight.121717DS1). Recipient BALB/c mice receiving TrED demonstrated consistently less weight loss within the first 3 weeks after transplant compared with recipients treated with cyclophosphamide on days 3 and 4 (Figure 1A). Additionally, overall clinical GVHD scores were lower in TrED- versus PTCy-treated recipients early after HSCT (Figure 1B). Long-term monitoring of recipients indicated that there was no significant difference between PTCy and TrED recipients in weight loss, clinical score, or overall survival (Figure 1, A-C). Notably, similar results were obtained using a different (lower) dosage of PTCy in this same HSCT model (Supplemental Figure 2).

Three weeks after transplant, the frequency and cell numbers of donor Tregs in the spleen as well as in blood were significantly higher in recipients of TrED (Figure 1D and Supplemental Figure 3A). PTCy-treated recipients also contained greater levels of donor Tregs compared with recipients of non-TrED without PTCy treatment (Figure 1D). We also examined colons of recipients and observed that total Treg levels both by percentage and numbers - were increased in TrED- versus PTCy-treated animals (Figure 1E). It is noteworthy that at this time point virtually all the peripheral Tregs ( $>90 \%-95 \%)$ were derived from the donor $\mathrm{T}$ cell inoculum and, therefore, $\mathrm{CD}^{+} \mathrm{RFP}^{+}$. Overall, these levels of Tregs after transplant correlated with the patterns of weight loss and clinical scores in groups of HSCT recipients. Histological assessment of colonic biopsies 3 weeks (Figure 1F) after HSCT illustrated that tissue from untreated HSCT recipients revealed severe colitis reflected by disruption of architecture, acute inflammation with severe lymphocyte infiltration, edema, mucosal thickening, and severe necrosis. In contrast, PTCy colonic tissue showed mild hyperplasia, mild inflammation, and edema in the submucosa. Finally, colon tissue from TrED recipients exhibited patchy and mild colitis without hyperplasia or necrosis. 

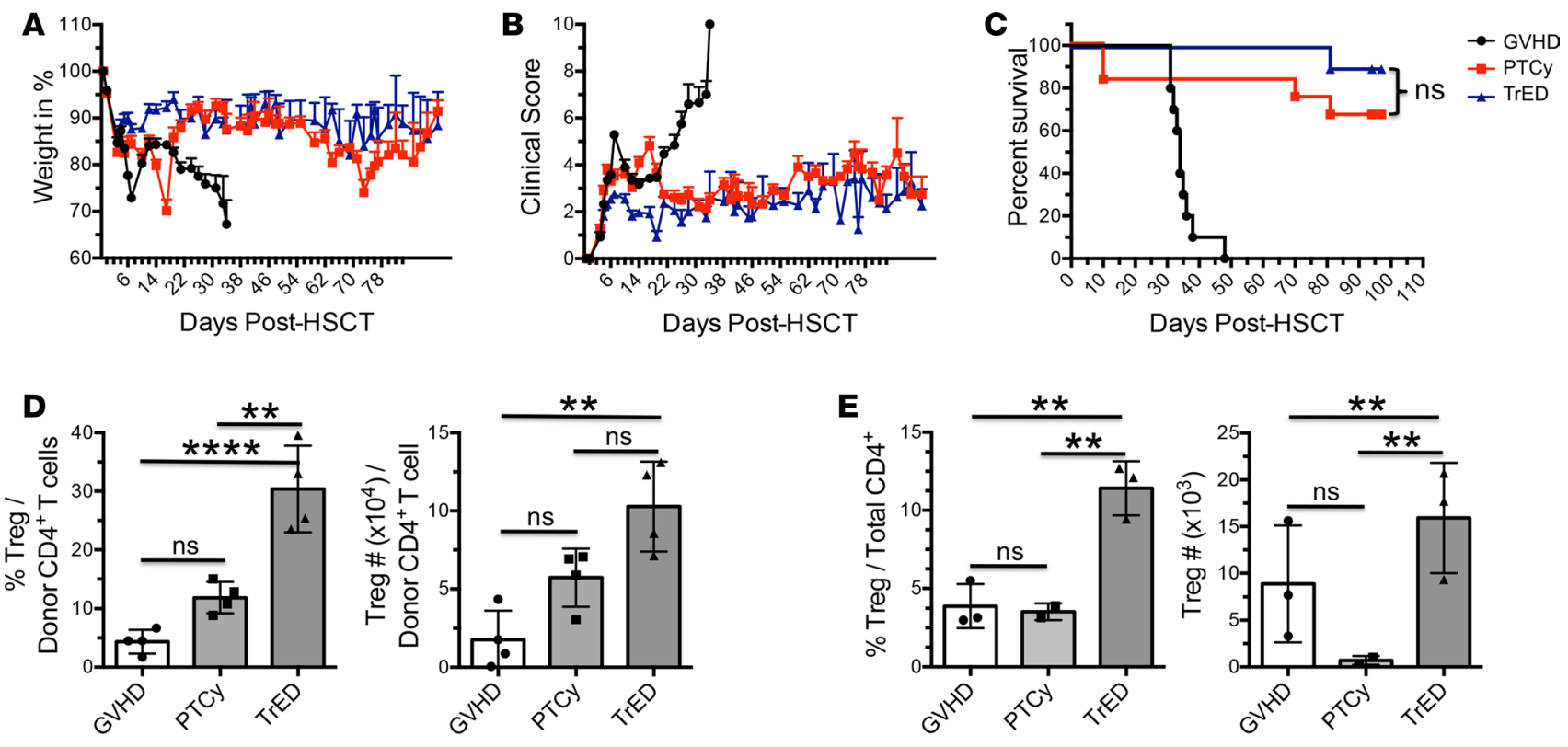

$\mathbf{F}$

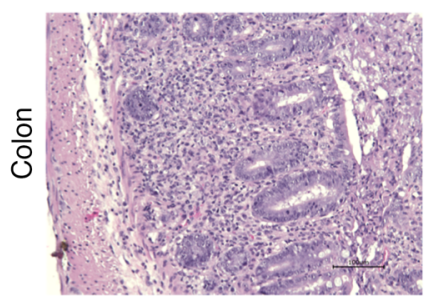

GVHD

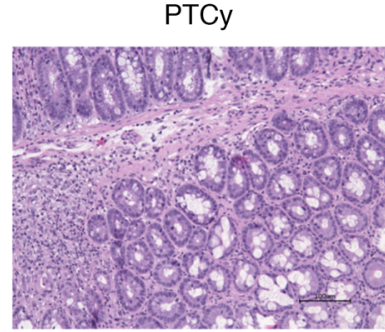

TrED

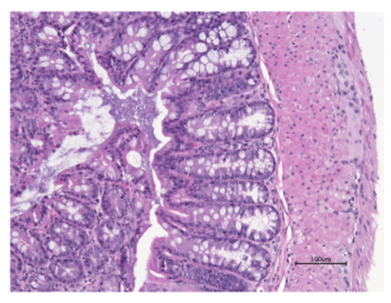

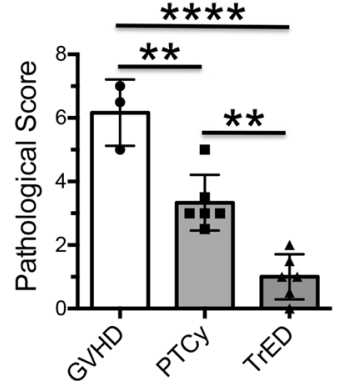

Figure 1. Treg-expanded donor cells show advantages over PTCy treatment early after transplant; however, long-term outcomes are comparable in a major MHC-mismatch model of preclinical HSCT. (A-C) A HSCT utilizing a B6 BALB/c donor/recipient mouse model involving a complete MHC mismatch was performed on day 0. Lethally irradiated (8.5-Cy) BALB/C mice received $5 \times 10^{6}$ TCD B6-CD45.1 BM cells and spleen cells from expanded (TL1A-Ig/IL-2; Treg-expanded donor cells [TrED] group) or untreated B6-FoxP3 ${ }^{\text {ffp }}$ (GVHD and PTCy group) donor mice adjusted to contain $1.1 \times 10^{6}$ total T cells. Cyclophosphamide was given on day 3 and 4 after HSCT at $80 \mathrm{mg} / \mathrm{kg}$ i.p. Weights (day 12-19) (A), clinical scores (day 12-19) (B), and survival ( $P$ = ns) (C) $(n=10-12)$. Statistical analysis for weights and clinical score was also performed to assess the overall model using JMP 13 Pro. No statistical significance between PTCy and TrED was detected. A log-rank test was used for survival analysis. (D) Donor Treg frequencies and cell numbers in spleens on day 21 after HSCT are significantly higher in TrED recipients compared with PTCy-treated and CVHD control animals $(n=4)$. Data are expressed as mean \pm SEM and were analyzed by ANOVA with Bonferroni correction for multiple comparisons. Data are pooled from 2 independent experiments. (E) Treg frequencies and cell numbers in lamina propria on day 21 after HSCT are significantly higher in TrED recipients compared with PTCy-treated and CVHD control animals $(n=3)$. Data are expressed as mean \pm SEM and were analyzed by ANOVA with Bonferroni correction for multiple comparisons. Data shown are from 1 experiment. (F) Representative H\&E-stained sections from colons on day 21 after HSCT show severe colitis in tissue from untreated HSCT recipients, with disruption of architecture, acute inflammation with severe lymphocyte infiltration, edema, mucosal thickening, and severe necrosis. In contrast, PTC colonic tissue showed mild hyperplasia, mild inflammation, and edema in the submucosa. Finally, colon tissue from TrED recipients exhibited patchy and mild colitis without hyperplasia or necrosis. Original magnification, $\times 200$. Pathology scores are shown on the right $(n=3-6)$. Data (from 2 independent experiments) are shown as mean \pm SEM; ANOVA with Bonferroni correction was applied for multiple comparisons. ${ }^{*} P<0.05 ;{ }^{* *} P<0.01 ;{ }^{* *} P<0.001 ;{ }^{* * *} P<0.0001$. Scale bars: $100 \mu \mathrm{m}$.

To extend these highly reproducible results in this MHC-mismatched transplant model, a second independent HSCT model involving MHC-matched, minor transplantation antigen-mismatched donors and recipients was examined (Figure 2). Following transplantation of TrED B10.D2 (H2d) donor T cells into $\mathrm{BALB} / \mathrm{c}\left(\mathrm{H}_{2}{ }^{\mathrm{d}}\right)$ recipients, virtually identical results to those after the MHC-mismatched transplant were observed early after HSCT, i.e., TrED-transplanted mice demonstrated less weight loss and lower clinical scores compared with those of PTCy-treated animals (Figure 2, A and B), and these differences reached statistical significance. Additionally, the overall survival was significantly higher in TrED- compared with PTCy-treated animals (Figure 2C). Histological analysis of the skin from recipient mice indicated that untreated recipient tissue exhibited substantial fibrosis and thickening, accompanied by moderate infiltration and inflammation with patchy necrosis (Figure 2D). However, skin from PTC-treated recipients 

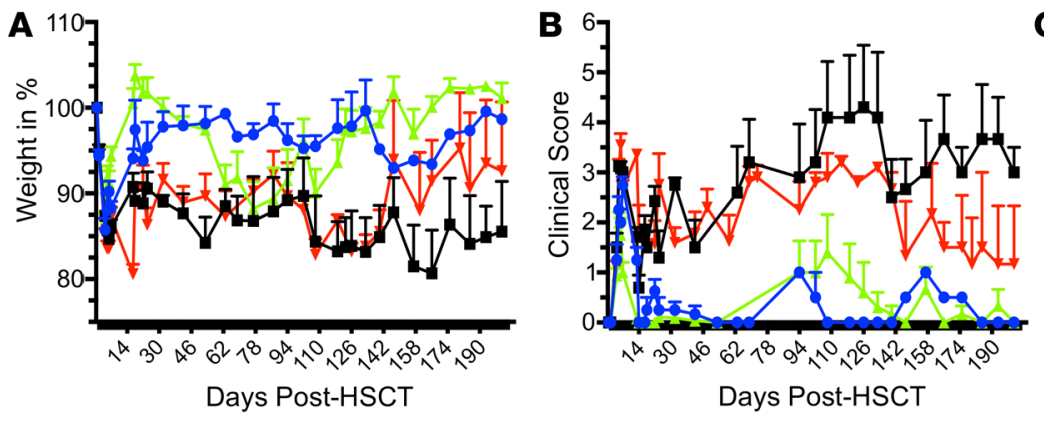

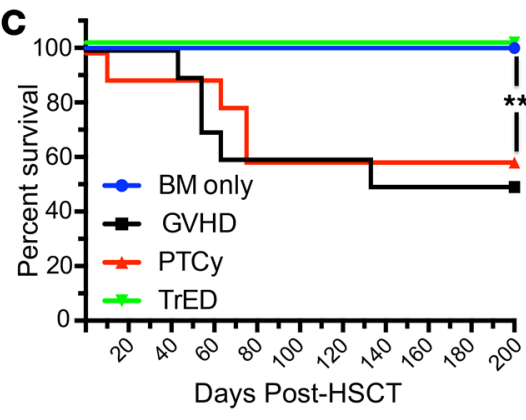

D

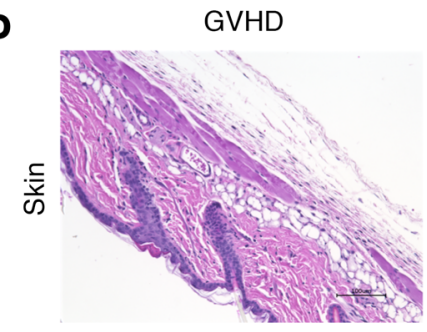

E

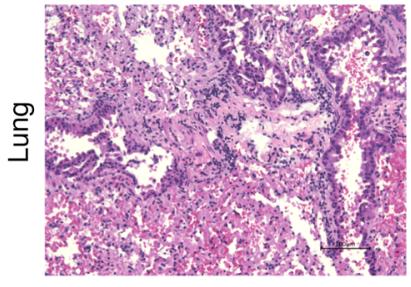

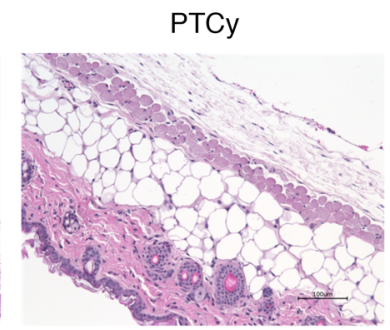

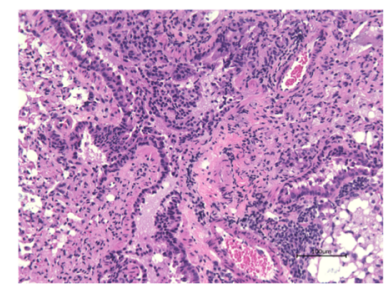

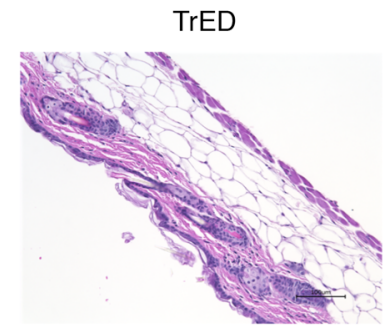

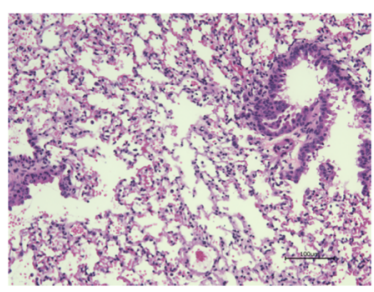

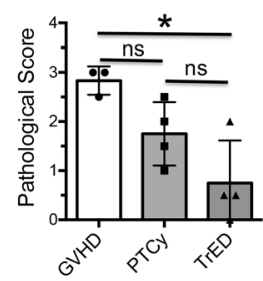

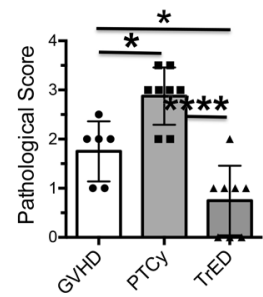

Figure 2. Comparison of TrED and PTCy treatment in a minor MHC-mismatch model shows comparable results to the major MHC-mismatch model. (A-C) A HSCT utilizing a B10.D2 BALB/c donor/recipient mouse model across a MHC-matched, minor histocompatibility antigen mismatch was performed using $8 \times 10^{6}$ non-TCD BM cells $+25 \times 10^{6}$ spleen cells from either untreated (CVHD and PTCy group) or TL1A-Ig/IL-2-expanded (TrED group) B10.D2 donor mice (BM: $n=8$; GVHD and PTCy: $n=12$; TrED: $n=17$ ). Percentage of initial weight (day 10-24, $P<0.0001)(\mathbf{A})$, clinical score (day 10-24, $P<0.0001)(\mathbf{B})$ and survival $\left.{ }^{* *} P<0.01\right)($ C) are presented. Survival was analyzed by log-rank test. Statistical analysis for weights and clinical score was also performed to assess the overall model using JMP 13 Pro. $P<0.05$ for all the groups. (D) Representative H\&E staining from untreated recipient skin on day 31 after HSCT exhibited substantial fibrosis and thickening accompanied by moderate infiltration and inflammation with patchy necrosis. However, skin from PTC-treated recipients showed mild fibrosis with mild inflammation and some infiltrate and hyperplasia. In contrast, no fibrosis or thickening was observed with only mild inflammation in TrED recipients. Original magnification, $\times 200$. Pathology scores are shown on the right $(n=3-4)$. Data are shown as mean \pm SEM; ANOVA with Bonferroni correction was applied for multiple comparisons. ${ }^{*} P<0.05$. Data are pooled from 2 independent experiments. Scale bars: 100 $\mu \mathrm{m}$. (E) Representative H\&E staining (chosen from 2 independent experiments) from recipient lung on day 200 after HSCT exhibited multifocal areas of moderate chronic, active inflammation, and fibrosis. The inflammation was characterized mainly by lymphocytes and macrophages with smaller numbers of neutrophils. Many alveoli also contained large macrophages and foamy cytoplasm in the PTCy compared with the TrED group, which was within normal limits. Original magnification, $\times 200$. Pathology scores are shown on the right $(n=6-8)$. Data are shown as mean \pm SEM; ANOVA with Bonferroni correction was applied for multiple comparisons. ${ }^{*} P<0.05 ;{ }^{* *} P<0.01$; ${ }^{* *} P<0.001 ;{ }^{* * *} P<0.0001$. Scale bars: $100 \mu \mathrm{m}$.

showed mild fibrosis with mild inflammation and some infiltrate and hyperplasia. In contrast, no fibrosis or thickening was observed, with only mild inflammation, in TrED recipients. Similarly, the GI tract was examined on day 31 in this matched model, and we observed infiltrates and inflammation in the colons of PTCy-treated recipients compared with little change in TrED recipients (data not shown). Notably, lung tissue was also examined $>6$ months after HSCT (Figure 2E). The tissue pathology of untreated mice undergoing GVHD included a marginal infiltration and fibrosis. Interestingly, infiltrates and fibrosis were also readily observed in the PTCy-treated recipients, whereas few alterations were observed in the longterm survivors receiving TrED transplants (Figure 2E and Supplemental Figure 4).

Functional immunity is intact in HSCT recipients receiving TrED or treated with PTCy. To assess the functional immunity in TrED- or PTCy-treated hematopoietic stem cell transplanted recipients, we investigated if tolerance to donor and recipient antigens and concomitant ability to reject third-party allografts was present in both groups of recipients 3 months after HSCT, when 100\% lethality had occurred in untreated animals. Two heterotopic skin grafts were applied to the trunk of each mouse, one from B6 $\times$ BALB/c F1 (H2 $\left.{ }^{b / d}\right)$ mice and one from $\mathrm{C} 3 \mathrm{H} / \mathrm{HeJ}$ third-party $\left(\mathrm{H} 2^{\mathrm{k}}\right)$ donors (Figure 3). Notably, all mice accepted the F1 skin grafts, 
A

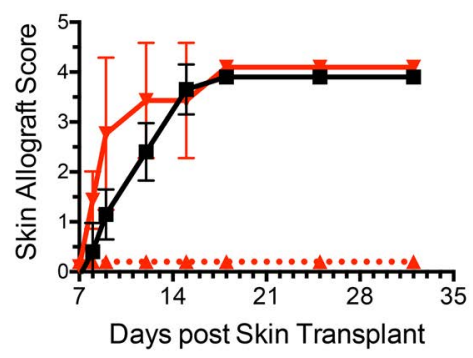

C
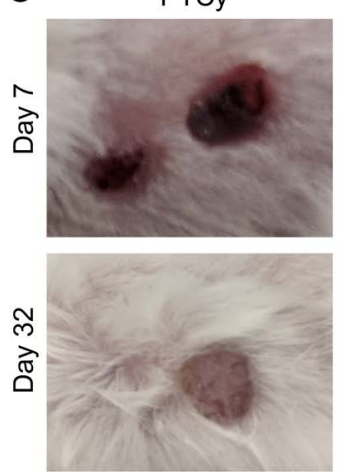

B

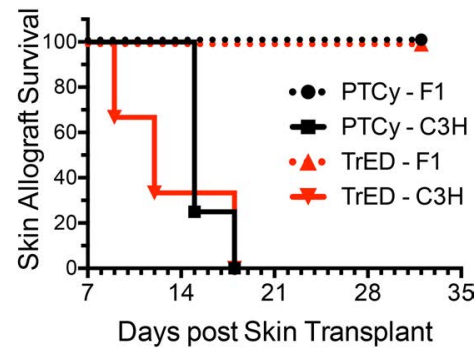

TrED
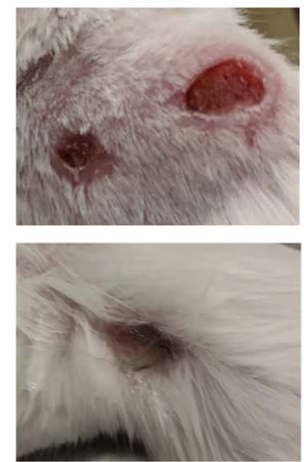

Figure 3. Functional immunity is intact in TrED recipients and PTCy-treated animals. TrED and PTCy recipients received 2 skin grafts, applied on the trunk of each mouse, 1 from B6xBALB/C F1 ( $\left.\mathrm{H}^{\mathrm{b} / \mathrm{d}}\right)$ mice and 1 from $\left.\mathrm{C} 3 \mathrm{H} / \mathrm{He}\right)$ third-party $\left(\mathrm{H} 2^{\mathrm{k}}\right.$ ) donors 3 months after HSCT. Grafts were assessed and scored on days 7, 8, 9, 12, 15, 18, 25, and 32 ( $n$ =4). (A) Allograft score. Graft scoring was performed as follows: 0, intact graft and healthy appearance; 1, inflamed graft, but without signs of necrosis observed; 2 , inflamed graft and less than $25 \%$ necrosis observed; 3 , inflamed graft and between $25 \%$ and $75 \%$ necrosis observed; and 4 , greater than $75 \%$ necrosis detected or loss of graft. (B) Allograft survival. All mice accepted the F1 (B6×BALB/c) skin grafts, whereas all C3H/HeJ grafts were rejected in both PTCy and TrED transplant recipients by day 18. (C) Representative photographs of skin grafts present on recipient mice on days 7 and 32 from both groups (PTCy, left; TrED, right).

indicating that tolerance was present in both donor and host alloantigens (Figure 3A). In contrast, all C3H/ HeJ grafts were rejected in both groups by day 18 (Figure 3B). These results suggest that, at this time after transplant, $\mathrm{T}$ cell immune reconstitution was sufficient to enable generation of first set allograft rejection responses in both groups of HSCT recipients.

Recipients of either TrED or PTCy treatment were also examined for their ability to generate $\mathrm{T}$ cell recall responses following priming against alloantigens. Recipients were therefore injected twice (14 days apart) with $\mathrm{C} 3 \mathrm{H} / \mathrm{HeJ}$ spleen, lymph node (LN), and thymus cells 3 months after transplant (Figure 4A). Four days following the second immunization, animals received a challenge cell dose containing CFSE-labeled spleen cells from both $\mathrm{C} 3 \mathrm{H} / \mathrm{HeJ}$ and $\mathrm{B} 6 \times \mathrm{BALB} / \mathrm{c} \mathrm{F} 1\left(\mathrm{H} 2^{\mathrm{b} / \mathrm{d}}\right)$ mice. Cytotoxicity was determined 18 hours later by relative levels of $\mathrm{H} 2^{\mathrm{k}}$ versus $\mathrm{H} 2^{\mathrm{b} / \mathrm{d}}$ target cells via flow cytometric analysis. Although no killing was detected in unprimed transplant recipients, both TrED and PTCy groups showed comparable levels of killing against $\mathrm{H} 2^{\mathrm{k}}$ target cells (Figure 4). Notably, neither group demonstrated killing against target cells expressing self (i.e., donor $\mathrm{H} 2^{\mathrm{b}}$ or recipient $\mathrm{H} 2^{\mathrm{d}}$ ) antigens (Figure $4 \mathrm{~B}$ ).

$A$ more rapid lymphoid engraftment is seen in TrED compared with PTCy recipients. Following transplant, experiments were performed to assess the origin of lymphoid cells present in recipients of both $\operatorname{TrED}$ and PTCy treatment from 1-3 months after HSCT. To determine if cells in the peripheral blood were derived from mature donor $\mathrm{T}$ cells, transplanted hematopoietic progenitors, or surviving recipient lymphocytes, flow analysis was performed after staining with anti-CD45.1 and anti- $\mathrm{K}^{\mathrm{b}} \mathrm{mAbs}$ (Figure 5). After the first month after HSCT, TrED recipients had the highest levels of donor bone marrow-derived (BM-derived) (CD45 $\mathrm{H}_{2} \mathrm{~K}^{\mathrm{b}}$ ) $\mathrm{CD}^{+} \mathrm{CD}^{-}$and $\mathrm{CD}^{-} \mathrm{CD}^{+} \mathrm{T}$ cells (Figure 5) as well as Tregs (Supplemental Figure 3B). By 2 months after HSCT, T cell engraftment was comparable in the two groups, and, by 3 months, both TrED and PTCy recipients were virtually indistinguishable (Figure 5). These latter findings are consistent with the clinical scores and survival of both groups but support the notion that there is greater de novo lymphopoiesis 


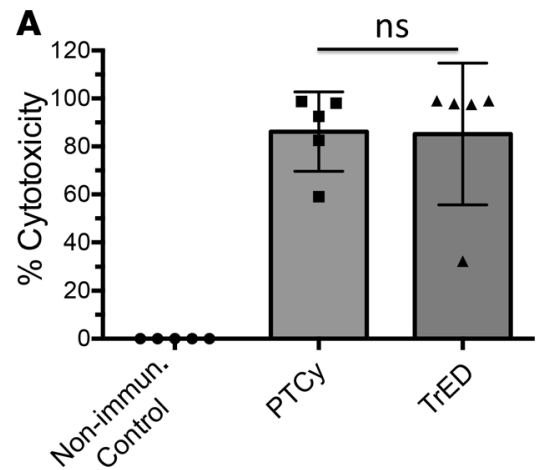

B Non-immun.

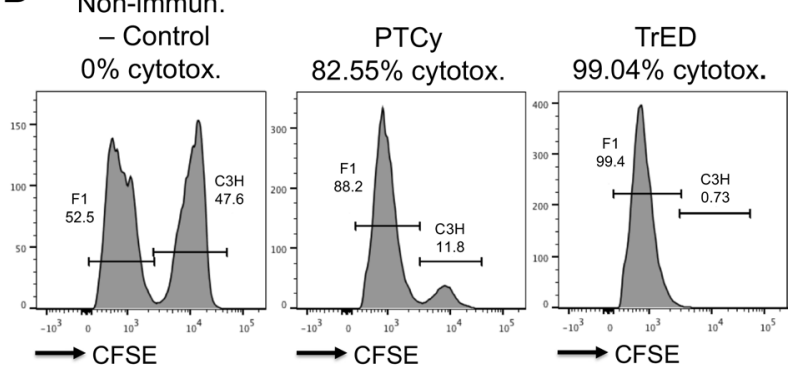

Figure 4. aHSCT recipients of both TrED and PTCy are able to mount $\mathrm{T}$ cell recall responses following priming against alloantigens. Three months after HSCT, recipients were immunized twice, 14 days apart, with $50 \times 10^{6}$ spleen, LN, and thymic cells from third-party complete MHC-disparate C3H/ $\mathrm{HeJ}$ mice $\left(\mathrm{H} 2^{\mathrm{k}}\right)$. Four days after the last immunization, splenocytes from both third-party $\mathrm{C} 3 \mathrm{H} / \mathrm{HeJ}\left(\mathrm{H} 2^{\mathrm{k}}\right)$ and $\mathrm{F} 1$ cells $\left(\mathrm{BALB} / \mathrm{C} \times\left[57 / B \mathrm{C}\right.\right.$; $\left.\mathrm{H} 2^{\mathrm{b} / \mathrm{d}}\right)$ were labeled with high $(5 \mu \mathrm{M})$ and low $(0.5 \mu \mathrm{M})$ levels of CFSE, respectively, and inoculated at a ratio of 1:1 $\left(20 \times 10^{6}\right.$ total) into groups of (a) unimmunized BM-transplanted BALB/c ("control") mice and the immunized mice from both (b) TrED- and (c) PTCy-treated recipients. 12-18 hours later, all mice were sacrificed and cytotoxicity was assessed in the spleen by gating on the CFSE ${ }^{+}$cells $(n=5)$. Cytotoxicity was calculated using the following formula: $1-\left(\mathrm{C} \mathrm{H}^{\text {sample }} \times \mathrm{F} 1^{\text {control }}\right) /\left(\mathrm{C} \mathrm{H}^{\text {control }} \times \mathrm{F} 1^{\text {sample }}\right) \times 100$. (A) TrED and PTCy recipients show comparable anti-C3H/HeJ H2 ${ }^{\mathrm{k}}$ third-party cytotoxicity. Data are expressed as mean \pm SEM and were analyzed by a 2-tailed unpaired $t$ test. Data are pooled from 2 independent experiments. (B) Representative flow cytometry histograms. Lower fluorescent peaks of CFSE-labeled C3H versus F1 cells indicate cytotoxicity against these third-party target cells.

derived from donor HSCs in mice undergoing TrED versus PTCy transplant treatment. Consistent with these observations, we detected greater levels of naive CD4 T cells for the first 2 months and naive CD8 $\mathrm{T}$ cells for the first 3 months (Supplemental Figure 5). We also observed that $>90 \%$ of $\mathrm{CD} 11 \mathrm{~b}^{+} \mathrm{Gr}-1^{+}$cells, which may include MDSCs at 1 month in all transplant groups, were derived from the donor marrow consistent with the rapid recovery of myeloid cells 2-4 weeks after transplant (data not shown).

Superior recovery of thymopoiesis in TrED-compared with PTCy-treated HSCT recipients. Three weeks following MHC-mismatched HSCT in TrED- or PTCy-treated recipients, thymic tissues were first analyzed for total cell numbers and $\mathrm{T}$ cell subsets including, $\mathrm{CD} 4^{+} \mathrm{FoxP} 3^{+}$Tregs. Significantly, greater overall numbers of thymocytes (5-10 times) were consistently identified in recipients of TrED- compared with PTCy-treated or control animals (Figure 6A). The first and earliest sign of thymic injury in animal models and in humans is loss of intermediate $\mathrm{CD} 4^{+} \mathrm{CD} 8^{+}$double-positive (DP) thymocytes. Notably, phenotypic assessment of DP thymocytes and more mature single-positive (SP) cells demonstrated that TrED recipients had relatively normal levels of $\mathrm{CD} 4^{+} \mathrm{CD} 8^{-}$and $\mathrm{CD} 4^{-} \mathrm{CD} 8^{+} \mathrm{SP}$ cells as well as DP thymocytes (Figure $6 \mathrm{~B}$ ). PTCy-treated recipients contained few DP thymocytes and a preponderance of SP cells, indicating thymic injury and impaired thymopoietic recovery (Figure 6B). This pattern of injury and dysregulation of thymopoiesis was similar to that seen in untreated animals with clinical GVHD, which is known to directly effect primary and secondary lymphoid tissues, including the thymus (Figure 6B).

Because of the differences in overall thymocyte numbers, the relative frequency of thymic progenitor cells, i.e., CD4-CD8 ${ }^{-}$double-negative (DN) cells, was greater in PTCy-treated and untreated HSCT recipients compared with those treated with TrED (Figure 6B). Assessment of the DN CD44/CD25 subsets present at 3 weeks found no significant difference between the $4 \mathrm{DN}$ subsets in TrED- and PTCy-treated recipients, although there was a trend toward increased DN4 (CD44-CD25-) cells, but it was not significant (Supplemental Figure 6A). Examination of DN subsets approximately 1 week later showed a statistically significant increase in DN4 and a decrease in DN3 (CD44-CD25+) subsets in TrED recipients versus PTCy-treated HSCT mice (Supplemental Figure 6B). In summary, differences were observed in both progenitor and developing thymocyte populations between HSCT recipients of TrED or PTCy treatment.

Next, the origin of SP-developing thymocytes was examined (Figure 6C). Low levels (<20\%) CD45.1 $1^{+}$thymocytes derived from transplanted donor progenitor cells were identified in tissue from untreated or PTCy-treated recipients (Figure 6C). In contrast, assessment of thymocytes in TrED recipients demonstrated much greater levels ( $60 \%-80 \%$ of all SP cells) of donor stem cell-derived CD $45.1^{+}$thymocytes, consistent with the higher overall thymocyte numbers and normal SP/DP phenotype of these tissues. Interestingly, examination of Treg numbers and frequency in the thymuses of all transplanted groups indicated that the highest numbers and 
Day 30
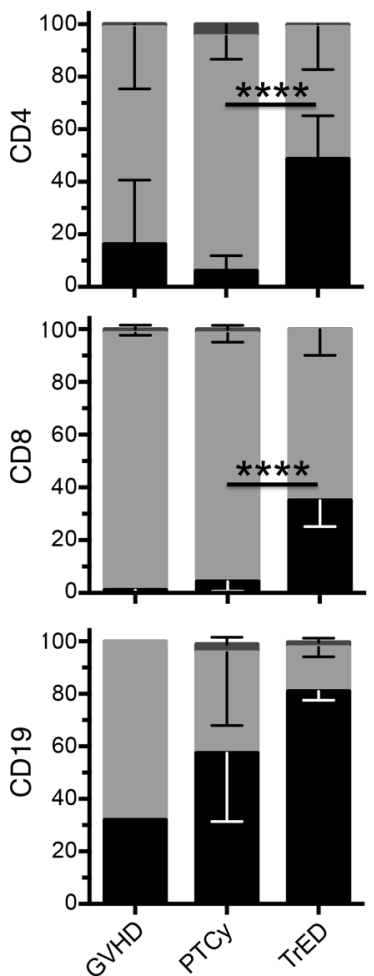

Day 60
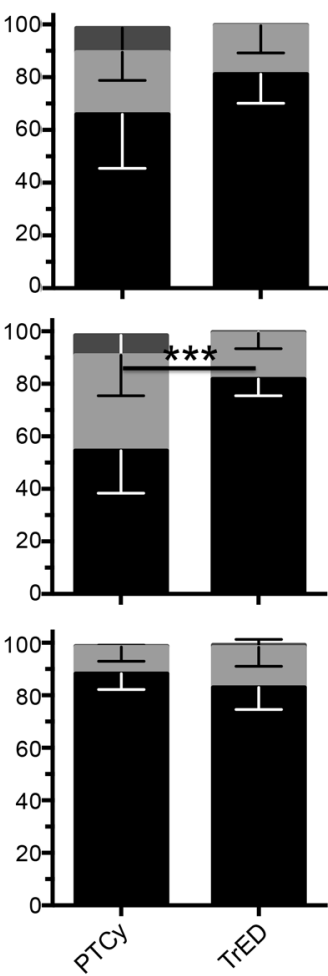

Day 90
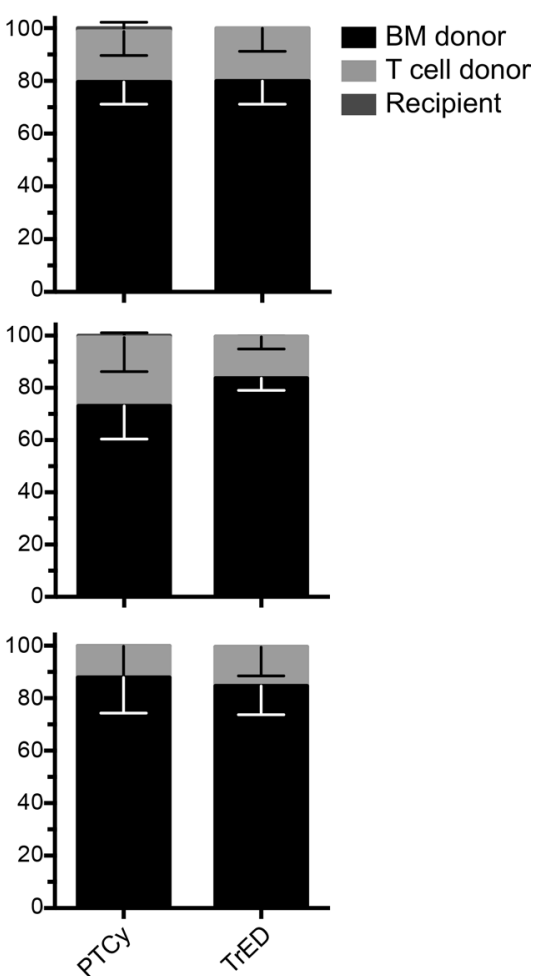

Figure 5. More rapid peripheral engraftment in TrED recipients compared with PTCy-treated animals. The origin of lymphoid cells from transplanted mature donor T cells (CD45.1, $\mathrm{H} 2 \mathrm{~K}^{\mathrm{b}+}$ ), transplanted hematopoietic progenitors ( $\mathrm{T}$ celldepleted bone marrow $=\left(\mathrm{CD} 45.1^{+}, \mathrm{H} 2 \mathrm{~K}^{\mathrm{b}+}\right)$, or surviving recipient lymphocytes (host: $\left.\mathrm{CD} 45.1^{-}, \mathrm{H} 2 \mathrm{~K}^{\mathrm{b}}\right)$, present in recipients of TrED and PTCy was assessed in peripheral blood by flow cytometry from 1-3 months after HSCT by staining with directly conjugated anti-CD45.1 and anti-K $\mathrm{K}^{\mathrm{b}}$ mAbs. A significantly faster engraftment of CD4 and CD8 cells was detected in TrED recipients compared with PTCy-treated and CVHD controls (first column, day 30). CVHD, $n=3$; PTCy and TrED, $n=$ 8-10. Data are expressed as mean $\pm \mathrm{SEM}$ and were analyzed by a 2-tailed unpaired $t$ test. ${ }^{* * *} P<0.001 ;{ }^{* * *} P<0.0001$.

frequencies of $\mathrm{CD}^{+} \mathrm{FoxP}^{+} / \mathrm{CD}^{+}$were found in the TrED HSCT recipients (Figure 6D). Notably, these Tregs were derived from mature donor $\mathrm{T}$ cells contained in the transplant and not produced by the recipient thymus.

These results confirm that early thymic function is superior in recipients of TrED versus PTCy-treated or untreated HSCT recipients. These findings are consistent with the engraftment results, demonstrating that peripheral blood of TrED recipients contained significantly higher levels of donor stem cell-derived $\mathrm{T}$ cells within the first month after HSCT (Figure 5). We therefore hypothesized that the generation of recent thymic emigrants (RTEs) early after HSCT would depend on whether recipients were transplanted with TrED or received PTCy treatment. To address this question, BALB/c mice were transplanted using B6-RAG2p-GFPmismatched donor BM and B6-WT splenic T cells (Figure 7). Peripheral blood from groups of mice was examined 3-4 weeks after HSCT (Figure 7 and Supplemental Figure 7A). As anticipated RTEs were readily identified in recipients receiving BM alone without development of GVHD and were marginally detectable in mice undergoing (i.e., untreated) GVHD (Figure 7A). Importantly, there was virtually no difference in the levels of both $\mathrm{CD}^{+}$and $\mathrm{CD} 8^{+}$RTE populations in PTCy-treated recipients compared with untreated GVHD mice (Figure 7A). However, $\mathrm{CD} 4^{+}$and $\mathrm{CD} 8^{+} \mathrm{RTE}$ were readily identified and markedly different in $\mathrm{TrED}$ recipient blood compared with those in PTCy or GVHD mice $(P>0.001)$ (Figure 7$)$. Notably, examination of $\mathrm{CD} 19^{+}$recent marrow emigrant B cells followed a similar pattern to the RTE T cell observations. While PTCy-treated recipients did contain significant levels of recent marrow emigrants B cells, TrED recipients contained a significantly higher frequency of this population, which was comparable to that present after transplantation of BM alone (Figure 7A, right). It is important to point out that no differences were detected in the RTE levels present between PTCy and TrED recipients 2 months after HSCT (Supplemental Figure 7B). In total, the findings are consistent with the presence of enhanced thymic function early after transplant resulting in elevated RTE production and higher levels of lymphoid engraftment in recipients of TrED. 
A

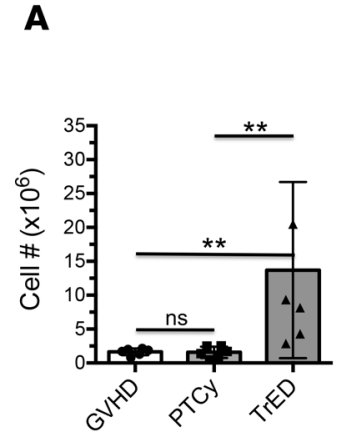

B
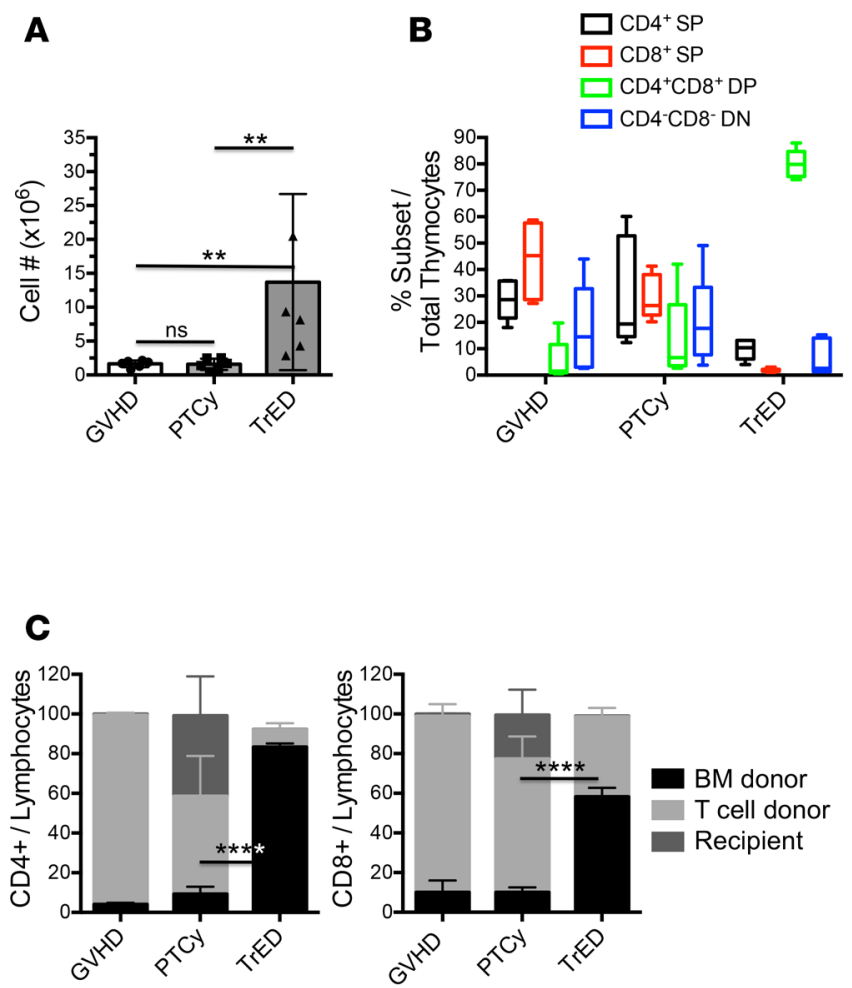
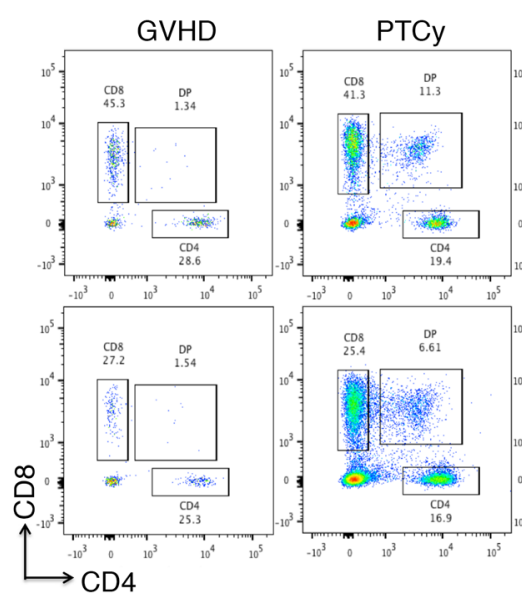

D

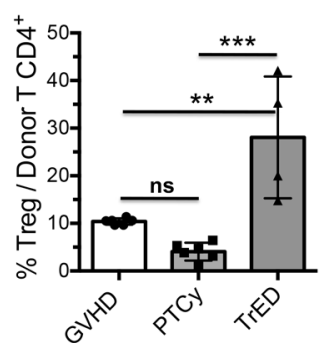

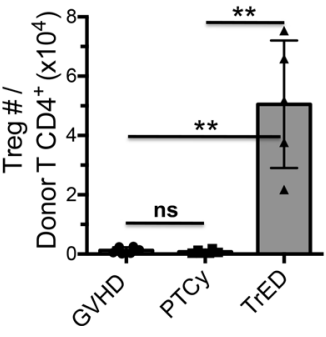

Figure 6. Faster thymic recovery in TrED recipients compared with PTCy-treated animals and GVHD controls early after HSCT. Three weeks after HSCT, thymic tissue was analyzed for total cell numbers (A), phenotype (B), engraftment (C), and Tregs $(\mathbf{D})(n=4-6)$. (A) Significantly greater cell numbers were identified in TrED recipients compared with PTCy-treated and control animals. Data from 3 pooled independent experiments are shown as mean \pm SEM; ANOVA with Bonferroni correction was applied for multiple comparisons. (B) Normal levels of CD4+CD8- and CD4-CD8+ SP as well as DP thymocytes are detected in TrED recipients. PTC-treated animals and GVHD controls show an abnormal pattern of thymocytes, with few DP cells and a prevalence of SP cells (left). Representative dot plots for each group are shown (right). (C) Assessment of the origin of developing thymocytes shows a significantly faster engraftment from donor CD45.1 $1^{+}$stem cells in the CD4+ and CD8 ${ }^{+}$SP compartment in TrED recipients compared with PTCy-treated animals and CVHD controls. Data are expressed as mean \pm SEM and were analyzed by a 2 -tailed unpaired $t$ test. Data are pooled from 2 independent experiments. (D) TrED recipients exhibit higher frequencies and numbers of donor $\mathrm{CD}^{+}{ }^{+} \mathrm{Foxp}^{+}$Tregs. Data from 3 pooled independent experiments are shown as mean $\pm \mathrm{SEM}$; ANOVA with Bonferroni correction was applied for multiple comparisons. ${ }^{* *} P<0.01 ;{ }^{* *} P<0.001 ;{ }^{* * *} P<0.0001$.

\section{Discussion}

The administration of PTCy early after HSCT is an extremely effective GVHD prophylaxis strategy and has promoted a dramatic expansion of haploidentical and mismatched clinical aHSCT $(4,5,30)$. More recently, the administration of Tregs with transplantation of donor $\mathrm{T}$ cells has also shown promise as a strategy to ameliorate development of acute $\operatorname{GVHD}(11,13,31)$. The purpose of the present studies was to utilize well-characterized models of preclinical aHSCT to directly compare the PTCy and TrED approach with regard to inhibition of GVHD and the effect of these strategies on the recovery of thymopoiesis and a functional $\mathrm{T}$ cell repertoire.

Our studies demonstrated that long-term weight loss and clinical scores were not significantly different between groups of recipients treated with PTCy or transplanted with TrED. However, we found that TrED-transplanted recipients exhibited less weight loss and lower clinical scores in the critical period early after HSCT, when NRM is most likely to occur. Additionally, initial peripheral lymphoid engraftment derived from transplanted BM stem/progenitor cells was higher in TrED- versus PTCy-treated recipients. Consistent with this observation, thymocyte phenotype as well as this tissue's function following TrED transplant was better - as evidenced by virtually normal levels of SP and DP thymocytes and the presence of greater levels of RTEs derived from donor BM progenitor cells during the first month after HSCT.

Following aHSCT, patients experience increased rates of infection due to neutropenia and delays in recovery of quantitative and functional lymphoid immunity (32-34). This immune impairment occurs in part as a consequence of slow lymphoid reconstitution, which can delay effective antiviral and other immune responses for months or years following aHSCT (35-39). Such delay can result from damage occurring from conditioning as well as alloreactive GVH responses, which injure central as well as peripheral immune 
A

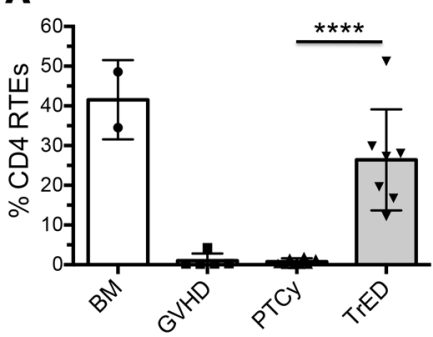

CD8

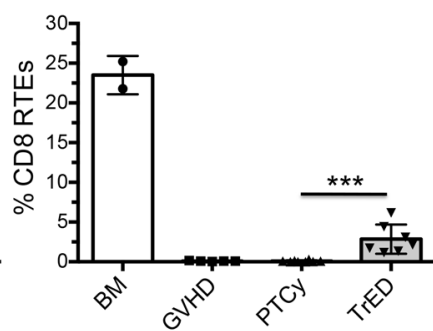

CD19

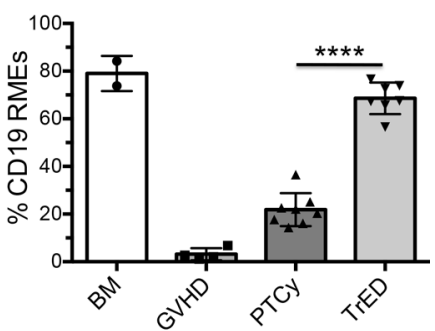

\section{B}
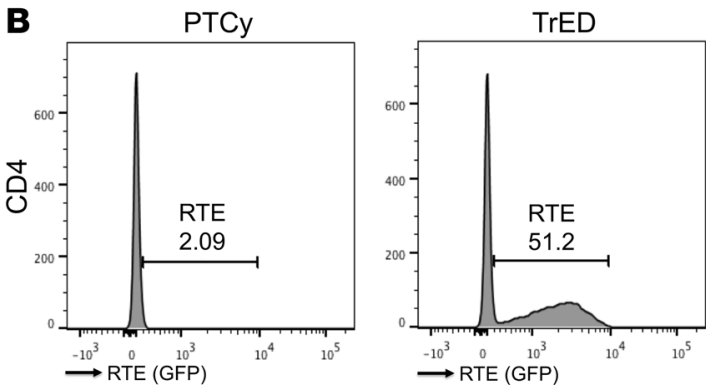

Figure 7. Significantly higher frequencies of recent thymic/marrow emigrants in TrED recipients versus PTCy-treated animals and CVHD controls early after HSCT. A HSCT utilizing a B6 BALB/C donor/recipient mouse model involving a complete MHC mismatch was performed on day 0 . Lethally irradiated (8.5-Gy) BALB/c mice received $5 \times 10^{6}$ TCD B6-RAC2p-GFP BM cells and spleen cells from expanded (TL1A-Ig/IL-2; TrED group) or untreated B6-FoxP3 ${ }^{\text {rfp }}$ (GVHD and PTCy group) donor mice adjusted to contain $1.1 \times 10^{6}$ total T cells. Cyclophosphamide was given on day 3 and 4 after $\mathrm{HSCT}$ at $80 \mathrm{mg} / \mathrm{kg}$ i.p. Recent thymic/marrow emigrants (RTEs/RMEs) were analyzed in PB by flow cytometry 3 weeks after HSCT (BM, $n=2$; GVHD, $n=5$; PTCy and TrED, $n=8$ ). (A) Significantly higher frequencies of CD4+ and CD8 ${ }^{+}$RTEs as well as CD19+ $B$ cells (RMEs) are detected in TrED recipients compared with PTCy-treated animals and CVHD controls. Data are expressed as mean \pm SEM and were analyzed by a 2-tailed unpaired $t$ test. (B) A representative histogram from the TrED and the PTCy group is shown. ${ }^{*} P<0.05 ;{ }^{* *} P<0.01$; ${ }^{* *} P<0.001 ;{ }^{* * *} P<0.0001$.

compartments $(28,33,40)$. The overall immunocompromised state in patients promotes bacterial and other microbial infections, and, together with GVHD, these complications are responsible for the largest portion of morbidity in nonrelapsed transplant recipients as well as patients transplanted for nonmalignant disorders (41). Accordingly, important objectives of strategies to alleviate posttransplant complications are to reduce transplant-related mortality, prevent GVHD, and promote efficient immune reconstitution and engraftment.

Comorbidities present in HSCT recipients at the time of transplant are associated with decreased overall survival due to increased rates of nonrelapse mortality (NRM), including adverse outcomes associated with acute and chronic GVHD (42-44). Thus, GVHD prophylaxis strategies that minimize additional systemic toxicities in the early posttransplant period should provide clinical benefits by lowering NRM risks after aHSCT. During the first 7-10 days after transplant, recipients of TrED but not PTCy treatment demonstrated less weight loss and lower clinical scores compared with untreated HSCT recipients. One potential clinical benefit would be to decrease risks of early GI toxicities that in humans could lead to increased rates of bacterial translocation of the gut, which contributes to the risk of bacterial sepsis and NRM.

Notably, we observed significantly greater levels of lymphoid engraftment present in peripheral tissues in TrED- compared with PTC-treated recipients. Accordingly, we hypothesized that thymic function was stronger in TrED recipients. In contrast to untreated or PTCy-treated recipients, the relative frequency of SP and DP (>65\%) thymocyte populations was virtually normal in TrED recipients, and overall cell numbers were also greater in these animals. To more directly assess thymic function in these groups of transplant recipients, levels of RTEs were assessed, and significantly higher levels of RTEs were detected in the periphery of TrED recipients, correlating with the observed thymocyte phenotypic profiles. Hence, we anticipate that immune function at this time point will be more effective in TrED recipients. Less thymic damage could be a consequence of greater suppression of alloreactive donor anti-host $\mathrm{T}$ cells as well as direct effects of cyclophosphamide within the thymic compartment (45-47). Such activity could involve damage to stromal cells, including cortical and medullary epithelium that are vital to normal thymocyte differentiation, and studies are currently in progress to examine such a mechanism (47). Although there is clearly a difference 
Table 1. Outcomes following allogeneic HSCT comparing untreated recipients with recipients treated with PTCy or the use of TrED

\begin{tabular}{|c|c|c|}
\hline First 3-4 weeks after HSCT & PTCy treatment days 3-4 & TrED transplant \\
\hline Early body weight & Decreased & Increased \\
\hline Early clinical score & Increased & Decreased \\
\hline Thymic damage & No difference & Decreased \\
\hline RTEs & No difference & Increased \\
\hline Engraftment & No difference & Increased \\
\hline Overall survival & Increased & Increased \\
\hline
\end{tabular}

in these preclinical transplants regarding lymphoid reconstitution early after HSCT, it is important to point out that survivors of PTCy and TrED treatment both demonstrated effective overall lymphoid reconstitution and acceptance of donor and recipient skin grafts, concomitant with rejection of third-party complete mismatched tissue allografts. Hence, tolerance to donor and recipient self-antigens as well as effective in vivo immune responsiveness was evident in both groups of long-term survivors. These findings indicate that functional thymic reconstitution does occur (we did not detect differences in thymocyte numbers at later time points, day 60; data not shown) - albeit more slowly in PTCy-treated recipients. It may be notable that, although B cells were not extensively examined here, overall CD19+ levels in the blood and spleen including $\mathrm{CD}_{19}{ }^{+}$recent marrow emigrants - were also higher in TrED versus PTCy recipients. Thus, taken together with the above findings, the observations support the notion that the use of TrED resulted in less overall damage to central, i.e., thymus and BM, lymphoid compartments compared with the use of day 3 and 4 high-dose cyclophosphamide in mice receiving myeloablative HSCT. Autoreactive T cells have been posited to contribute to chronic GVHD (cGVHD), and abnormal lymphoid differentiation may contribute to development of such T cells $(48,49)$. Therefore, although cGVHD was not evaluated in the present studies, it is possible that establishment of earlier normal immune $\mathrm{T}$ and $\mathrm{B}$ differentiation and reconstitution after allo-HSCT may diminish development of such autoreactive lymphoid cells and decrease cGVHD. It may therefore be noteworthy that, although TGF- $\beta$ is a major effector molecule of Tregs, in contrast to PTCy-treated animals, recipients of TrED transplants did not exhibit fibrosis in lung tissue 6-7 months after HSCT (Figure 2 and Supplemental Figure 4).

It may be interesting to consider our results in the context of more cell-based versus more cytokine-based GVHD. Thus, investigating the outcomes of PTCy and TrED in transplants following lower intensity conditioning could be informative with regard to the relative effectiveness of these treatment strategies. For example, it is possible that differences in cytokine levels could result in different levels of donor anti-host alloreactive T cells. However, to date several reports have not identified differences in PTCy effectiveness with respect to engraftment and transplant outcomes after varying levels of conditioning in haploidentical HSCT $(50,51)$.

Finally, the mechanism of action of these two strategies differ remarkably, i.e., chemo-induced deletion of conventional cells (by PTCy) versus suppression of conventional lymphocytes and APC (by Tregs), and this likely underlies the findings of early differences between PTCy and TrED usage in the present experiments (see Table 1 summary). Nonetheless, in total, the findings demonstrate that the use of TrED in the experimental aHSCTs employed here was as effective as PTCy treatment, with regard to GVHD amelioration, and support the hypothesis that use of TrED may provide benefit for recipients early after transplant, with respect to transplant-related mortality and initial immune function. While we and others have investigated GVT following the use of donor Tregs $(10,52)$, it will be important in future investigations, to employ suitable tumors and a valid model to precisely assess and directly compare the effectiveness of both of the treatment strategies.

\section{Methods}

Mice. Wild-type BALB/c (H2 $\left.{ }^{\mathrm{d}}\right), \mathrm{B} 6 \times \mathrm{BALB} / \mathrm{c} \mathrm{F} 1\left(\mathrm{H} 2^{\mathrm{d}, \mathrm{b}}\right)$, and $\mathrm{C} 3 \mathrm{H} / \mathrm{HeJ}\left(\mathrm{C} 3 \mathrm{H} ; \mathrm{H} 2^{\mathrm{k}}\right)$ mice were purchased from Taconic or The Jackson Laboratory. B6-FoxP $3^{\text {rfp }}$ were provided by R. Flavell (Yale University, New Haven, Connecticut, USA) (53), B6-CD45.1 and B10.D2 (H2 $\left.{ }^{\mathrm{d}}\right)$ mice were bred in our facility. RAG2pGFP mice were provided by R. Tisch (University of North Carolina, Chapel Hill, North Carolina, USA) $(54,55)$. Mice were used at $6-12$ weeks of age and were maintained in pathogen-free conditions at the University of Miami animal facilities. 
Antibodies and reagents. Commercial antibodies for use in flow cytometry were purchased from BD Biosciences (CD25 [PC61); CD45.1 [A20]; H2K [AF6-88.5]), Biolegend (CD4 [RM4-5 or GK1.5]; CD8 [536.7]; CD19 [6D5]; CD44 [IM7]; Foxp3 [FJK-16s]) or eBioscience (CD62L [MEL-14]); and recombinant mouse IL-2 and $\alpha$-IL-2 mAb, clone JES6-5H4, were purchased from eBioscience. The IL-2/ $\alpha$-IL-2 complex was generated by incubating $1.5 \mu \mathrm{g}$ recombinant mouse IL- 2 with $8 \mu \mathrm{g}$ JES6-5H4 for 15 minutes at room temperature. The fusion protein TL1A-Ig was generated as described previously (56).

Flow cytometry. Single-cell suspensions were prepared from different organs (spleen, LN, BM, colon). Peripheral blood was collected in heparinized tubes. PBMCs were isolated by standard Ficoll density gradient centrifugation. $10^{6}$ cells were preblocked with anti-mouse CD16/CD32 and stained with different antibody combinations. Intracellular staining was performed according to standard procedures. Flow cytometric analysis was performed on a BD LSR Fortessa HTS instrument. DIVA or FlowJo software was used for analysis.

Histologic analysis and immunohistochemistry. Tissues from animals on day 21 (major), 31, and 200 (minor antigen) after HSCT were fixed in $10 \%$ formalin and embedded in paraffin. Sections were stained with $H \& E$ and Masson's trichrome (lung) for histologic examination. Images were acquired using the Keyence BZ-X700 microscope. Tissue samples were scored following a modified system described by D. Kaplan, et al. (57). In brief, multiple parameters were used to compare pathology scores between groups in the skin, lung, and the colon (3, 2, and 4 parameters, respectively).

HSCT experiments. For the HSCT in the major MHC-mismatch model (B6 BALB/c), female BALB/c mice $\left(\mathrm{H}_{2}{ }^{\mathrm{d}}\right)$ received ablative conditioning with 8.5-Gy total body irradiation 1 day prior to transplant. BM cells were obtained from femurs, tibias, and vertebrae from sex-matched B6-CD45.1 (H2 $2^{\text {; }}$ Thy 1.2) or RAG2p-GFP donor animals. A single-cell suspension of marrow cells was prepared by flushing bones with a 21 -gauge needle, and the cells were filtered through a $100-\mu$ m nylon mesh. T cell depletion (TCD) of donor marrow cells was achieved via complement-mediated lysis using anti-T cell-specific antibody HO-13-4 (hybridoma supernatant, mouse anti-Thy1.2 IgM; ATCC) provided by Bruce Blazar (University of Minnesota, Minneapolis, Minnesota, USA), anti-CD4 mAb (clone 72.4), anti-CD8 mAb (clone H02.2), and rabbit complement (Cedarlane Laboratories). The marrow cells were incubated at $37^{\circ} \mathrm{C}$ for 45 minutes, washed twice in RPMI, and resuspended for hematopoietic cell transplant. Marrow TCD was routinely $>99 \%$. Donor T cells were prepared from spleens obtained from B6-FoxP3 ${ }^{\text {rfp }}$ expanded or nonexpanded animals. Donor cells were stained for T cells (anti-CD4, clone RM4-5; anti-CD8, clone 53-6-7) and adjusted to $1.1 \times 10^{6} \mathrm{~T}$ cells per mouse prior to mixing with BM. Recipient mice were transplanted (day 0 ) with TCD BM $\left(5 \times 10^{6}\right)$ and $1.1 \times 10^{6} \mathrm{~T}$ cells i.v. in a $0.2-\mathrm{ml}$ volume via tail vein injection.

For the MHC-matched, minor antigen-mismatch model (B10.D2 BALB/c), lethally irradiated (8.0Gy) BALB/c mice were transplanted (day 0) with $8 \times 10^{6}$ non-TCD BM cells and $25 \times 10^{6}$ splenocytes from B10.D2 mice.

GVHD was assessed by monitoring recipients for changes in total body weight, clinical signs, and overall survival. The clinical signs of GVHD were recorded for individual mice. Recipients were scored on a scale from 0 to 2 for 5 clinical parameters (58): (a) weight loss; (b) diarrhea; (c) fur texture; (d) posture; and (e) alopecia.

Donor Treg expansion. TL1A-Ig $(50 \mu \mathrm{g})$ was administered i.p. on days $1-4 ;$ rmIL-2 $(1.5 \mu \mathrm{g})$ bound to $\alpha-\mathrm{IL}-2 \mathrm{mAb}$ (clone JES6-5H4; $8 \mu \mathrm{g}$ ) on days 4 and 6 . Mice were sacrificed on day 7 , and spleen cells were used as T cell source in HSCT (10).

Cyclophosphamide treatment. Cyclophosphamide was administered i.p. (80 or $50 \mathrm{mg} / \mathrm{kg}$ ) on day 3 and 4 after $\operatorname{HSCT}(4,59)$.

Skin transplant. Allograft dorsal ear skin from euthanized F1 (B6×BALB/c, MHC H-2 $\left.{ }^{\mathrm{b} / \mathrm{d}}\right)$ mice and $\mathrm{C} 3 \mathrm{H}\left(\mathrm{MHC} \mathrm{H}-2^{\mathrm{k}}\right)$ mice was transplanted onto a $0.5 \times 0.5 \mathrm{~cm}^{2}$ prepared left lateral thoracic cavity skin bed of an isoflurane-anesthetized BALB/c mice 3 month after B6 allogenic HSCT (MHC H-2 ${ }^{\text {b/d }}$ alleles). The F1 skin allografts were placed cephalad and ventral and the $\mathrm{C} 3 \mathrm{H}$ skin allografts caudal and dorsal at the prepared skin bed site. The site was covered with an adhesive bandage, which remained in place for 6 days. Allograft appearance and integrity was assessed subsequently on days 7, 8, 9, 12, 15, 18, 25, and 32. Graft scoring was as follows: 0 , intact graft and healthy appearance; 1 , inflamed graft, but no signs of necrosis; 2 , inflamed graft and less than 25\% necrosis; 3 , inflamed graft and between $25 \%$ and $75 \%$ necrosis; and 4 , greater than $75 \%$ necrosis or loss of graft (60).

In vivo cytotoxicity assay. Three months after HSCT, mice were immunized twice (14 days apart) with $50 \times$ $10^{6}$ third-party $\mathrm{C} 3 \mathrm{H}$ splenocytes, LN cells, and thymocytes. Four days after the last immunization, splenocytes from third-party $\mathrm{C} 3 \mathrm{H}$ and $\mathrm{F} 1$ cells $(\mathrm{BALB} / \mathrm{c} \times \mathrm{C} 57 / \mathrm{B} 16)$ were labeled with high $(5 \mu \mathrm{M})$ and low $(0.5 \mu \mathrm{M})$ levels 
of CFSE, respectively, and inoculated at a ratio of 1:1 (20 × $10^{6}$ total) in an unimmunized, BM-transplanted control mouse and the immunized surviving mice from the PTCy and Treg groups. 12-18 hours later the mice were sacrificed and cytotoxicity was assessed in the spleen by gating on the $\mathrm{CFSE}^{+}$cells. Cytotoxicity was cal-

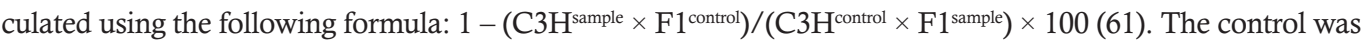
a nonimmunized BM-only-transplanted mouse in which no cytotoxicity of $\mathrm{C} 3 \mathrm{H}$ cells was happening.

Statistics. JMP 13 Pro (SAS) was used to compile the clinical scores and weights of the major and minor histocompatibility complexes. We plotted the means of the clinical scores and weights for each day and treatment group. Analysis was performed assessing the overall model. The overall model by least squares determined the regression of each treatment group, parameter estimates, and ANOVA.

All other statistical analysis was performed using GraphPad Prism. Values shown in graphs represent the mean of each group + SEM. Survival data were analyzed with the Mantel-Cox log-rank test. Nonparametric unpaired 2-tailed $t$ test was used for comparisons between 2 experimental groups, and multiple variable analysis was performed using ANOVA. A $P$ value less than 0.05 was considered significant.

Study approval. All experiments were performed in accordance with the Guide for the Care and Use of Laboratory Animals (National Academies Press, 2011), and the protocol was approved by the IACUC of the University of Miami Miller School of Medicine.

\section{Author contributions}

DW designed research studies, conducted experiments, analyzed data, and wrote the paper. CSB, HB, SC, BJP, and COL conducted experiments and acquired and analyzed data. NHA analyzed data regarding histopathology. RBL and KVK jointly supervised and supported the work and wrote the paper. RBL designed research studies.

\section{Acknowledgments}

We thank R. Tisch (University of North Carolina) for providing the RAG2p-GFP mice. We are grateful to J. Zaias and B. Fuentes for supporting the transfer of mice from University of North Carolina. We also thank P. Ruiz and Dayami Hernandez for their expert help with histology. This work was supported by funds from the Sylvester Comprehensive Cancer Center (to RBL and KVK), the NIH (RO1 EY024484-01 to RBL), and the Kalish Family Foundation (to KVK).

Address correspondence to: Robert B. Levy, University of Miami MSOM, PO Box 016960 (R138), Miami, Florida 33101, USA. Phone: 305.243.4542 or 305.243.6215; Email: rlevy@med.miami.edu.

1. Couriel D, Caldera H, Champlin R, Komanduri K. Acute graft-versus-host disease: pathophysiology, clinical manifestations, and management. Cancer. 2004;101(9):1936-1946.

2. Zeiser R, Blazar BR. Pathophysiology of chronic graft-versus-host disease and therapeutic targets. N Engl J Med. 2017;377(26):2565-2579.

3. Zeiser R, Blazar BR. Acute graft-versus-host disease - biologic process, prevention, and therapy. N Engl J Med. 2017;377(22):2167-2179.

4. Luznik L, Fuchs EJ. High-dose, post-transplantation cyclophosphamide to promote graft-host tolerance after allogeneic hematopoietic stem cell transplantation. Immunol Res. 2010;47(1-3):65-77.

5. Luznik L, O'Donnell PV, Fuchs EJ. Post-transplantation cyclophosphamide for tolerance induction in HLA-haploidentical bone marrow transplantation. Semin Oncol. 2012;39(6):683-693.

6. McCurdy SR, et al. Comparable composite endpoints after HLA-matched and HLA-haploidentical transplantation with post-transplantation cyclophosphamide. Haematologica. 2017;102(2):391-400.

7. Tang Q, Bluestone JA, Kang SM. CD4(+)Foxp3(+) regulatory T cell therapy in transplantation. J Mol Cell Biol. 2012;4(1):11-21.

8. Hanash AM, Levy RB. Donor CD4+CD25+ T cells promote engraftment and tolerance following MHC-mismatched hematopoietic cell transplantation. Blood. 2005;105(4):1828-1836.

9. Hoffmann P, Ermann J, Edinger M, Fathman CG, Strober S. Donor-type CD4(+)CD25(+) regulatory T cells suppress lethal acute graft-versus-host disease after allogeneic bone marrow transplantation. J Exp Med. 2002;196(3):389-399.

10. Wolf $\mathrm{D}$, et al. Marked in vivo donor regulatory T cell expansion via interleukin-2 and TL1A-Ig stimulation ameliorates graftversus-host disease but preserves graft-versus-leukemia in recipients after hematopoietic stem cell transplantation. Biol Blood Marrow Transplant. 2017;23(5):757-766.

11. Taylor PA, Lees CJ, Blazar BR. The infusion of ex vivo activated and expanded CD4(+)CD25(+) immune regulatory cells inhibits graft-versus-host disease lethality. Blood. 2002;99(10):3493-3499.

12. Trzonkowski $P$, et al. First-in-man clinical results of the treatment of patients with graft versus host disease with human ex vivo expanded CD4+CD25+CD127- T regulatory cells. Clin Immunol. 2009;133(1):22-26.

13. Brunstein CG, et al. Infusion of ex vivo expanded T regulatory cells in adults transplanted with umbilical cord blood: safety 
profile and detection kinetics. Blood. 2011;117(3):1061-1070.

14. Golovina TN, Mikheeva T, Brusko TM, Blazar BR, Bluestone JA, Riley JL. Retinoic acid and rapamycin differentially affect and synergistically promote the ex vivo expansion of natural human T regulatory cells. PLoS One. 2011;6(1):e15868

15. Copsel S, et al. Very Low Numbers of CD4(+) FoxP3(+) Tregs expanded in donors via TL1A-Ig low-dose IL-2 exhibit a distinct activation/functional profile suppress GVHD in a preclinical model [published online ahead of print May 8, 2018.]. Biol Blood Marrow Transplant. https://doi.org/10.1016/j.bbmt.2018.04.026.

16. Matsuoka KI. Low-dose interleukin-2 as a modulator of Treg homeostasis after HSCT: current understanding and future perspectives. Int J Hematol. 2018;107(2):130-137.

17. Ahlmann M, Hempel G. The effect of cyclophosphamide on the immune system: implications for clinical cancer therapy. Cancer Chemother Pharmacol. 2016;78(4):661-671.

18. Retière $\mathrm{C}$, et al. Impact on early outcomes and immune reconstitution of high-dose post-transplant cyclophosphamide vs anti-thymocyte globulin after reduced intensity conditioning peripheral blood stem cell allogeneic transplantation. Oncotarget. 2018;9(14):11451-11464.

19. Sportès C, et al. Establishing a platform for immunotherapy: clinical outcome and study of immune reconstitution after highdose chemotherapy with progenitor cell support in breast cancer patients. Biol Blood Marrow Transplant. 2005;11(6):472-483.

20. Farge D, et al. Long-term immune reconstitution and $\mathrm{T}$ cell repertoire analysis after autologous hematopoietic stem cell transplantation in systemic sclerosis patients. J Hematol Oncol. 2017;10(1):21.

21. Bashey A, et al. T-cell-replete HLA-haploidentical hematopoietic transplantation for hematologic malignancies using post-transplantation cyclophosphamide results in outcomes equivalent to those of contemporaneous HLA-matched related and unrelated donor transplantation. J Clin Oncol. 2013;31(10):1310-1316.

22. Kanakry CG, et al. Multi-institutional study of post-transplantation cyclophosphamide as single-agent graft-versus-host disease prophylaxis after allogeneic bone marrow transplantation using myeloablative busulfan and fludarabine conditioning. $J$ Clin Oncol. 2014;32(31):3497-3505.

23. Al-Homsi AS, Roy TS, Cole K, Feng Y, Duffner U. Post-transplant high-dose cyclophosphamide for the prevention of graft-versus-host disease. Biol Blood Marrow Transplant. 2015;21(4):604-611.

24. Kanakry CG, et al. Low immunosuppressive burden after HLA-matched related or unrelated BMT using posttransplantation cyclophosphamide. Blood. 2017;129(10):1389-1393.

25. Mackall CL, Gress RE. Pathways of T-cell regeneration in mice and humans: implications for bone marrow transplantation and immunotherapy. Immunol Rev. 1997;157:61-72.

26. Douek DC, et al. Changes in thymic function with age and during the treatment of HIV infection. Nature. 1998;396(6712):690-695.

27. Poulin JF, et al. Direct evidence for thymic function in adult humans. J Exp Med. 1999;190(4):479-486.

28. Komanduri KV, et al. Delayed immune reconstitution after cord blood transplantation is characterized by impaired thymopoiesis and late memory T-cell skewing. Blood. 2007;110(13):4543-4551.

29. Parikh SH, et al. A novel reduced-intensity conditioning regimen for unrelated umbilical cord blood transplantation in children with nonmalignant diseases. Biol Blood Marrow Transplant. 2014;20(3):326-336.

30. Luznik L, et al. High-dose cyclophosphamide as single-agent, short-course prophylaxis of graft-versus-host disease. Blood. 2010;115(16):3224-3230.

31. Komanduri KV, Champlin RE. Can Treg therapy prevent GVHD? Blood. 2011;117(3):751-752.

32. Atilla E, Atilla PA, Bozda冈 SC, Demirer T. A review of infectious complications after haploidentical hematopoietic stem cell transplantations. Infection. 2017;45(4):403-411.

33. Ogonek J, et al. Immune reconstitution after allogeneic hematopoietic stem cell transplantation. Front Immunol. $2016 ; 7: 507$.

34. Mehta RS, Rezvani K. Immune reconstitution post allogeneic transplant and the impact of immune recovery on the risk of infection. Virulence. 2016;7(8):901-916

35. Parkman R. Antigen-specific immunity following hematopoietic stem cell transplantation. Blood Cells Mol Dis. 2008;40(1):91-93

36. Gress RE, Emerson SG, Drobyski WR. Immune reconstitution: how it should work, what's broken, and why it matters. Biol Blood Marrow Transplant. 2010;16(1 Suppl):S133-S137.

37. Komanduri KV. Thymic function and allogeneic T-cell responses in stem-cell transplantation. Cytotherapy. 2002;4(4):333-342.

38. Gress RE, Komanduri KV, Einsele H, Cooper LJ. Lymphoid reconstruction and vaccines. Biol Blood Marrow Transplant. 2007;13(1 Suppl 1):17-22.

39. Gea-Banacloche J, et al. National Institutes of Health Hematopoietic Cell Transplantation Late Effects Initiative: The Immune Dysregulation and Pathobiology Working Group Report. Biol Blood Marrow Transplant. 2017;23(6):870-881.

40. Storek J, Witherspoon RP, Storb R. T cell reconstitution after bone marrow transplantation into adult patients does not resemble T cell development in early life. Bone Marrow Transplant. 1995;16(3):413-425.

41. D'Souza A, and Fretham C. Current Uses and Outcomes of Hematopoietic Cell Transplantation (HCT): CIBMTR Summary Slides. Center for International Blood \& Marrow Transplant Research. https://www.cibmtr.org/ReferenceCenter/SlidesReports/ SummarySlides/pages/index.aspx. Updated June 13, 2018. Accessed September 17, 2018.

42. Sorror ML, et al. Hematopoietic cell transplantation (HCT)-specific comorbidity index: a new tool for risk assessment before allogeneic HCT. Blood. 2005;106(8):2912-2919.

43. Sorror ML, et al. Prospective Validation of the Predictive Power of the Hematopoietic Cell Transplantation Comorbidity Index: A Center for International Blood and Marrow Transplant Research Study. Biol Blood Marrow Transplant. 2015;21(8):1479-1487.

44. Vaughn JE, et al. Pre-transplant comorbidity burden and post-transplant chronic graft-versus-host disease. Br J Haematol. 2015;171(3):411-416.

45. Nguyen $\mathrm{VH}$, et al. The impact of regulatory T cells on T-cell immunity following hematopoietic cell transplantation. Blood. 2008;111(2):945-953.

46. Gaidot A, et al. Immune reconstitution is preserved in hematopoietic stem cell transplantation coadministered with regulatory T cells for GVHD prevention. Blood. 2011;117(10):2975-2983.

47. Fletcher AL, et al. Ablation and regeneration of tolerance-inducing medullary thymic epithelial cells after cyclosporine, cyclophosphamide, and dexamethasone treatment. J Immunol. 2009;183(2):823-831. 
48. Wu T, et al. Thymic damage, impaired negative selection, and development of chronic graft-versus-host disease caused by donor CD4+ and CD8+ T cells. J Immunol. 2013;191(1):488-499.

49. Rangarajan $\mathrm{H}$, et al. Emergence of T cells that recognize nonpolymorphic antigens during graft-versus- host disease. Blood. 2012;119(26):6354-6364.

50. Berger M, et al. Feasibility and Outcome of Haploidentical Hematopoietic Stem Cell Transplantation with Post-Transplant High-Dose Cyclophosphamide for Children and Adolescents with Hematologic Malignancies: An AIEOP-GITMO Retrospective Multicenter Study. Biol Blood Marrow Transplant. 2016;22(5):902-909.

51. Robinson TM, O’Donnell PV, Fuchs EJ, Luznik L. Haploidentical bone marrow and stem cell transplantation: experience with post-transplantation cyclophosphamide. Semin Hematol. 2016;53(2):90-97.

52. Edinger M, et al. CD4+CD25+ regulatory T cells preserve graft-versus-tumor activity while inhibiting graft-versus-host disease after bone marrow transplantation. Nat Med. 2003;9(9):1144-1150.

53. Wan YY, Flavell RA. Identifying Foxp3-expressing suppressor T cells with a bicistronic reporter. Proc Natl Acad Sci USA 2005;102(14):5126-5131.

54. Boursalian TE, Golob J, Soper DM, Cooper CJ, Fink PJ. Continued maturation of thymic emigrants in the periphery. Nat Immunol. 2004;5(4):418-425.

55. Yu W, et al. Continued RAG expression in late stages of B cell development and no apparent re-induction after immunization. Nature. 1999;400(6745):682-687.

56. Khan SQ, Tsai MS, Schreiber TH, Wolf D, Deyev VV, Podack ER. Cloning, expression, and functional characterization of TL1A-Ig. J Immunol. 2013;190(4):1540-1550.

57. Kaplan DH, Anderson BE, McNiff JM, Jain D, Shlomchik MJ, Shlomchik WD. Target antigens determine graft-versus-host disease phenotype. J Immunol. 2004;173(9):5467-5475.

58. Cooke KR, et al. An experimental model of idiopathic pneumonia syndrome after bone marrow transplantation: I. The roles of minor $\mathrm{H}$ antigens and endotoxin. Blood. 1996;88(8):3230-3239.

59. Ross D, Jones M, Komanduri K, Levy RB. Antigen and lymphopenia-driven donor T cells are differentially diminished by post-transplantation administration of cyclophosphamide after hematopoietic cell transplantation. Biol Blood Marrow Transplant. 2013;19(10):1430-1438.

60. Beckmann J, Kurts C, Klebba I, Bayer B, Klempnauer J, Hoffmann MW. The role of CD30 in skin and heart allograft rejection in the mouse. Transplant Proc. 2001;33(1-2):140-141.

61. Quah BJ, Wijesundara DK, Ranasinghe C, Parish CR. Fluorescent target array T helper assay: a multiplex flow cytometry assay to measure antigen-specific CD4+ T cell-mediated B cell help in vivo. J Immunol Methods. 2013;387(1-2):181-190. 